\title{
Study of Water and Dimethylformamide Interaction by Computer Simulation
}

\author{
João Manuel Marques Cordeiro and Luiz Carlos Gomide Freitas ${ }^{\mathrm{a}}$ \\ Departamento de Física e Química, Faculdade de Engenharia de Ilha Solteira \\ - UNESP, CP 31 - 15385-000 - Ilha Solteira-SP, Brazil \\ a Departamento de Química Universidade Federal de São Carlos, \\ CP 676 - 13565-905 São Carlos-SP, Brazil
}

Z. Naturforsch. 54 a, 110-116 (1999); received November 19, 1998

\begin{abstract}
Monte Carlo simulations of water-dimethylformamide (DMF) mixtures were performed in the isothermal and isobaric ensemble at $298.15 \mathrm{~K}$ and $1 \mathrm{~atm}$. The intermolecular interaction energy was calculated using the classical 6-12 Lennard-Jones pairwise potential plus a Coulomb term. The TIP4P model was used for simulating water molecules, and a six-site model previously optimised by us was used to represent DMF. The potential energy for the water-DMF interaction was obtained via standard geometric combining rules using the original potential parameters for the pure liquids. The radial distribution functions calculated for water-DMF mixtures show well characterised hydrogen bonds between the oxygen site of DMF and hydrogen of water. A structureless correlation curve was observed for the interaction between the hydrogen site of the carbonyl group and the oxygen site of water. Hydration effects on the stabilisation of the DMF molecule in aqueous solution have been investigated using statistical perturbation theory. The results show that energetic changes involved in the hydration process are not strong enough to stabilise another configuration of DMF than the planar one.
\end{abstract}

Key words: Monte Carlo Simulation; Solvent Effects; Statistical Perturbation Theory; Hydration of Peptides.

\section{Introduction}

The investigation of hydration effects on the structure of biologically interesting molecules is an important contribution to understand the role of water on the behaviour of those molecules in biological media [1,2]. Theoretical contributions to this research field have been obtained with the reaction field theory, molecular dynamics and Monte Carlo simulation methods [1 - 3]. In the reaction field approach the environment is treated as a dielectric continuum and the interaction with the solute is calculated with methods derived from the Onsager formalism. In molecular dynamics and Monte Carlo methods, the solute-solvent interactions are modelled by discrete molecules interacting via a suitable energy surface. These methods are powerful tools to obtain detailed descriptions of structural, energetic and dynamical features of liquids, either pure or as mixtures, on a

Reprint requests to Dr. J. M. M. Cordeiro;

E-mail: cordeiro@fqm.feis.unesp.br. molecular level [4 - 6]. Recently we have undertaken a theoretical investigation on the liquid properties emphasising the liquid structure and the solute-solvent interaction in binary mixtures using Monte Carlo simulation [7 - 10]. In the present work we investigate the DMF-water interaction and the solvent effect on the stabilisation of the DMF molecule in aqueous medium. The importance of structural and thermodynamic studies of this molecule is related to the conformation of amide moieties in peptides. The structures of the amide-water solution and details on the hydrogen bonds formed have been discussed. Statistical Perturbation Theory (SPT) has been used to investigate the influence of hydration on the conformation of the amide in solution.

\subsection{Free Energy Calculations}

SPT [11] has been established as a valuable method for calculating the free energy of processes [12 - 14]. According to SPT, changes in the free energy on an arbitrary reaction coordinate can be treated as a pertur-

0932-0784 / 99 / 0200-0110 \$06.00 (c) Verlag der Zeitschrift für Naturforschung, Tübingen · www.znaturforsch.com

This work has been digitalized and published in 2013 by Verlag Zeitschrift für Naturforschung in cooperation with the Max Planck Society for the Advancement of Science under a Creative Commons Attribution-NoDerivs 3.0 Germany License. 
bation. This method has been shown to be very useful for calculating the free energy of solvation, and good agreement with the experimental results has been reported [12 - 15]. In the present work, the rotation of the O-C-N-C dihedral angle of the peptide bond was taken as a reaction coordinate, and the amide free energy of hydration was calculated as a function of the dihedral angle. The difference in the free energy between the reference and the perturbed state as a function of increments $\Delta \phi$ on the dihedral angle is given by

$$
\begin{aligned}
& G(\phi+\Delta \phi)-G(\phi)= \\
& \quad-k_{\mathrm{B}} T \ln \langle\exp \{-\beta[E(\phi+\Delta \phi)-E(\phi)]\}\rangle_{\phi},
\end{aligned}
$$

where,

$$
\begin{aligned}
E(\phi+\Delta \phi) & =\left(E_{\text {int }}+E_{\text {solv } / \text { solv }}+E_{\text {solt } / \text { solv }}\right)_{\phi+\Delta \phi}, \\
E(\phi) & =\left(E_{\text {int }}+E_{\text {solv } / \text { solv }}+E_{\text {solt } / \text { solv }}\right)_{\phi}
\end{aligned}
$$

and $\beta=1 / k_{\mathrm{B}} T$.

In these equations $E_{\text {int }}$ is the internal energy of the solute, that is a function of the dihedral angle $\phi$, $E_{\text {solv/solv }}$ is the solvent-solvent interaction energy, and $E_{\text {solt/solv }}$ is the interaction energy between solute and solvent molecules. $T$ and $k_{\mathrm{B}}$ are the temperature and the Boltzmann constant, respectively.

\section{Computational Details}

\subsection{Intermolecular Potential Function}

To determine the potential topology, the energy $E_{a b}$ between molecules $a$ and $b$ was given by a sum of Coulomb and Lennard-Jones potentials centred on the sites:

$$
E_{a b}=\sum_{i j}\left[A_{i j} / r_{i j}^{12}-B_{i j} / r_{i j}^{6}+q_{i} q_{j} / r_{i j}\right],
$$

where $r_{i j}$ is the distance between site $i$ in $a$ and site $j$ in $b$, and $q_{i}$ and $q_{j}$ are fractional point charges located on the $i$ and $j$ molecular sites (it has been assumed that the electrostatic contribution to the intermolecular potential is suitably represented by fractional charges located on the molecular sites). For each site $k$, the parameters $A_{k k}$ and $B_{k k}$ were given by $A_{k k}=4 \varepsilon_{k} \sigma_{k}^{12}$ and $B_{k k}=4 \varepsilon_{k} \sigma_{k}^{6}$, where $\varepsilon_{k}$ and $\sigma_{k}$ are the Lennard-Jones parameters for the $k^{\text {th }}$ site. Parameters $A_{i j}$ and $B_{i j}$ for a nondiagonal interaction $[i, j]$ were obtained using the geometric combining rules $A_{i j}=\left(A_{i i} A_{j j}\right)^{12}$ and $B_{i j}=\left(B_{i i} B_{j j}\right)^{12}$ [16]. This is a standard procedure used in this field. In a previous work we have shown that the difference between the results obtained using the above geometric combining rule and the well know Lorentz-Berthelot rule is negligible [7].

\subsection{Molecular Models}

The DMF molecule was modelled as a six-site rigid structure (the methyl group is a monatomic site) [9]. Therefore intramolecular relaxation effects are not considered. The water molecule was represented by the TIP4P potential developed by Jorgensen and co-workers [17]. The potential parameters $A_{i j}$ and $B_{i j}$ needed to calculate water-DMF interactions were obtained using the potential functions for the pure liquids and the geometric combining rules presented above. No further attempt was made to optimise them. This procedure is justified by previous results $[7,8$, $10,15]$. Therefore, the results for the water-DMF energetics obtained in our calculation are a further verification of the quality of potential functions obtained from geometric combining rules.

\subsection{Monte Carlo Simulations}

Statistical mechanics simulations were carried out in the NPT ensemble at $298 \mathrm{~K}$ and internal pressure $1 \mathrm{~atm}$, employing Metropolis importance sampling [18] and cubic box boundary conditions implemented in the DIADORIM program [19]. The DMF-water mixtures consisted of 256 molecules (the ratio between the number of water and DMF molecules was chosen to reproduce the appropriated molar fraction) while in the study of the DMF hydration the systems consisted of 250 molecules of water and 1 molecule of DMF. Starting from the initial monomer distribution, a new configuration was generated by randomly translating and rotating a randomly chosen molecule along Cartesian coordinates. As the calculations were carried out in the NPT ensemble, new configurations were also generated by probing the density of the liquid with volume changes. Ranges for translations and rotations of the monomers and volume changes were adjusted to yield an acceptance/trial ratio of about 0.45 for new configurations. After a volume movement, the coordinates of the centre of mass of all molecules in the reference box were scaled in the 
usual way [16]. In the calculation of the configurational energy using (2), a full intermolecular interaction was considered whenever any of the site-to-site distance $r_{i j}$ fell bellow a cut-off radius of $9.5 \AA$. In a recent work it was shown that the conditions (such as including or not long-range corrections) used to optimise a given set of potential parameters must be maintained in subsequent calculations using these potential parameters [20]. The reason is that the potential parameters are constrained by the methodology used in the optimisation process to reproduce some thermodynamic results. Therefore, compatibility could be spoiled using the same set of parameters in different conditions. The contributions of Lennard-Jones interactions beyond the cut-off radius were considered using the formalism presented by Allen and Tildesley [16]. In the method discussed by these authors the long range contribution from the Lennard-Jones potential is included by integrating the potential function from the cut-off radius to infinity, assuming that the pair correlation function is unitary. In previous papers we have found that the long-range contribution from the Lennard-Jones potential amounts to 1 to $2 \%$ of the total interaction energy [ $7-10]$. The long-range contribution from the Coulomb potential can be introduced using the reaction field formalism discussed by Newman [21]. For liquid simulations, the reaction field formalism (RF) may be superior to the Ewald sum correction in the sense that artificial long-range correlation is not introduced in liquid simulations by the RF formalism [16]. A detailed investigation of the influence of including long-range corrections using RF methodology on the values of thermodynamic properties obtained in liquid simulations has been performed in our research group [22]. As a general trend, it was found that the effects on thermodynamical and structural properties are very small. Therefore, long range corrections for coulombic interactions beyond the cut-off radius were not included in the present work. As discussed elsewhere, site-site pair correlation functions, thermodynamic properties and single particle dynamics of polar liquids appear to be quite insensitive to the long range forces in the system [5], which is in accord with results obtained by us using the RF formalism [22].

In the present work, each calculation started with an equilibration phase of $1.2 \mathrm{M}$ configurations followed by an average segment consisting of $12 \mathrm{M}$ configurations $\left(\mathrm{M}=10^{6}\right)$. Statistical uncertainties were calculated from separate averages over blocks of $2 \times 10^{5}$ configurations. The calculations were carried out on IBM RISC 6000 workstations.

\section{Results and Discussions}

\subsection{Energetic}

The water-DMF mixing process is exothermic for all concentrations, with a maximum value of $-0.5 \mathrm{kcal} / \mathrm{mol}$ liberated at the concentration of $33 \%$ in DMF [23]. The fact that this process is exothermic has been interpreted as a consequence of hydrogen bond formation between the carbonilic oxygen of the amide and the molecules of water [23], which have been confirmed by Raman spectroscopy [24]. Calculations on the water-water and water-amide interaction energy have shown that hydrogen bonds between water and amides are stronger than between water molecules [25]. Results for the excess enthalpy as a function of the water mole fraction obtained in the present work are compared with experimental data in Figure 1. The agreement between the theoretical and experimental results is very good, especially considering the many body nature of the system under investigation. The largest difference observed between theoretical and experimental results is about $0.15 \mathrm{kcal} / \mathrm{mol}$, which is a very small quantity for such

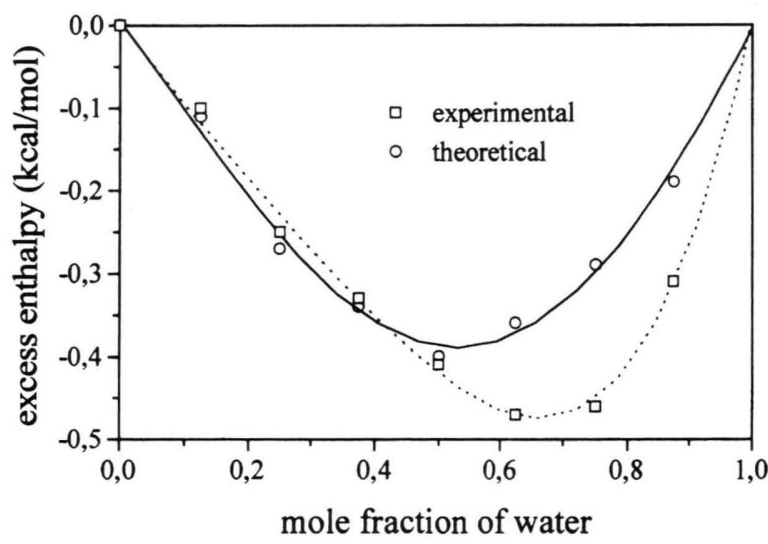

Fig. 1. Excess enthalpy $H^{\mathrm{E}}$ obtained for the DMF-water mixing process as a function of the water mole fraction. The excess enthalpy is calculated by $H^{\mathrm{E}}=\Delta H_{\mathrm{vap} \text {, mix }}-$ $\left(X_{1} \Delta H_{\text {vap }_{1}}+X_{2} \Delta H_{\text {vap }_{2}}\right.$ ), where $X_{i}$ is the molar fraction of liquid $i$ and $\Delta H_{\text {vap }}$ is the heat of vaporisation. In the heat of vaporisation is calculated by $\Delta H_{\text {vap }}=-E_{i}+R T$, where $E_{i}$ is the average energy per molecule obtained in the simulation and $R T=0.592 \mathrm{kcal} / \mathrm{mol}$ at $T=298 \mathrm{~K}$. Experimental results taken from [23]. 


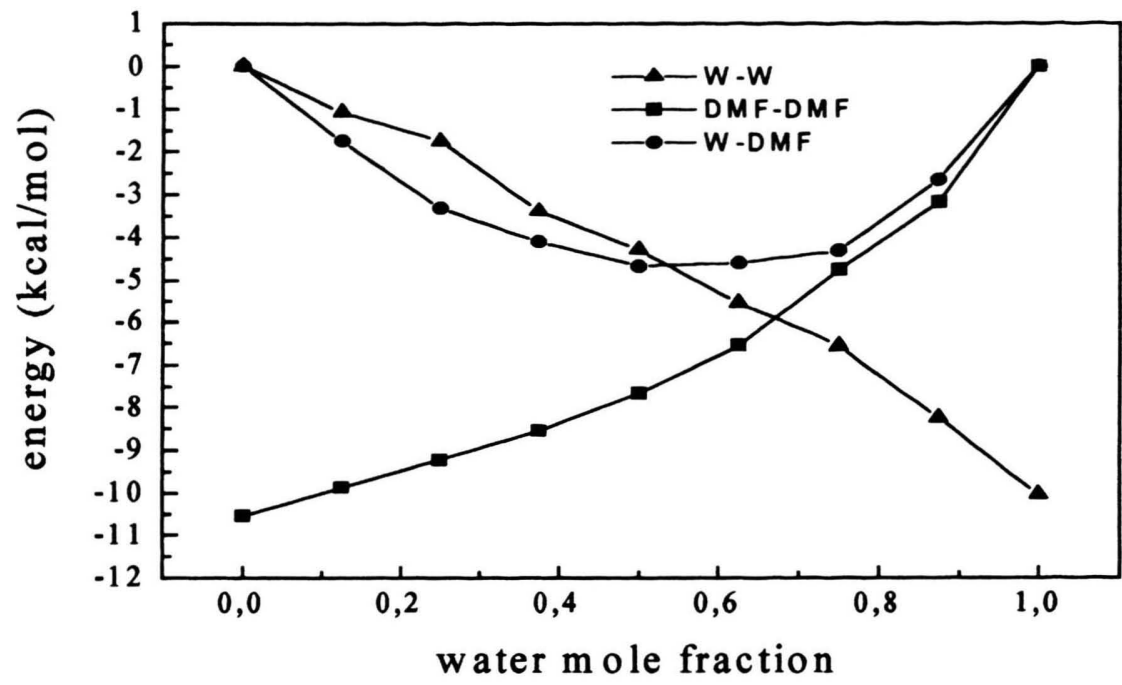

Fig. 2. Energies of interaction per molecule as a function of the water mole fraction.

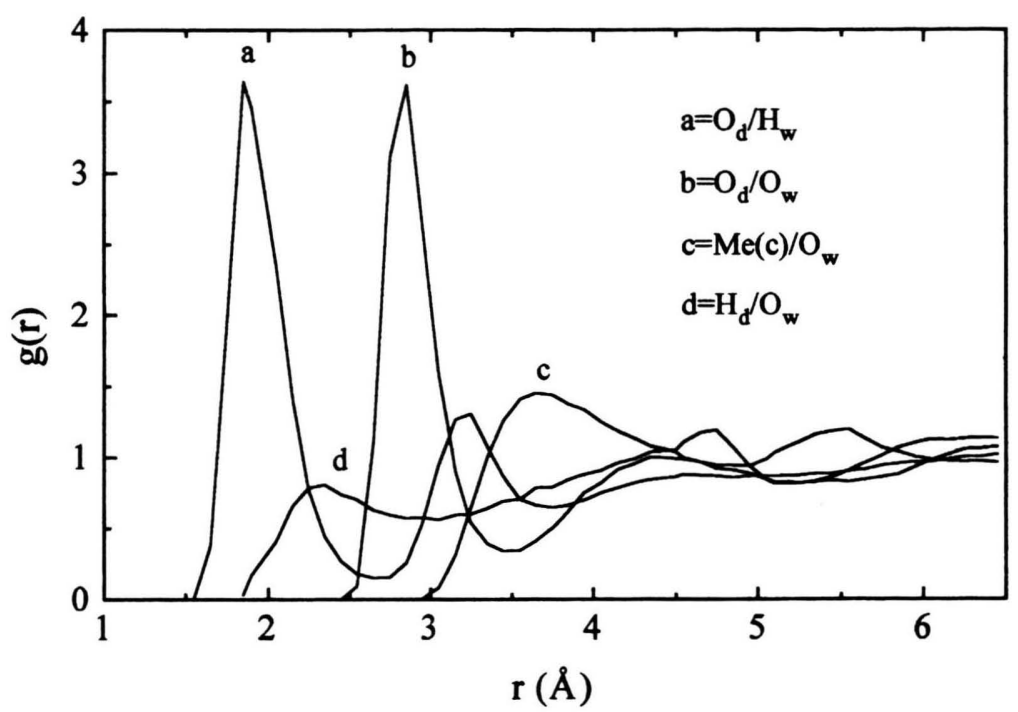

Fig. 3. Site-site radial distribution functions for water-DMF interaction in an equimolar mixture.

a system. In Fig. 1, worse agreement between calculated and experimental results is observed in the region of low concentration of DMF. In this region it is noticed that the energetic changes involved in the disruption of water-water hydrogen bonds and formation of water-DMF ones is very drastic. Therefore the potential functions used in the present work for waterDMF interactions need further adjustment to account for the energetics of this process. Similar behaviour was found in the study of hydration of amines [26]. In this paper it was found that the inclusion of a potential function to represent the solute polarisation was needed to reproduce the energetics of the hydration process. Improvements in the potential functions for water-DMF interaction can be pursued using the
SPT formalism to investigate the hydration energy of amides. However, since the potential functions for the water-DMF interaction were obtained from the pure liquids without further optimisation, the agreement observed in Fig. 1 reaffirms the validity of using combining rules to obtain potential parameters for cross interactions in liquid simulations.

In Fig. 2 a partitioning of the water-DMF interaction energy as a function of the mole fraction is presented, showing the contribution of each type of interaction to the total energy. It is worth noticing that this energy partitioning is a consequence of the pair additive approximation assumed in (2). As a general trend, the data for the interaction energies follow the pattern expected for a binary mixing process: the 
water-water interaction energy increases as the concentration of this component is raised, and a similar behaviour is noticed for the DMF-DMF interaction as a function of DMF concentration. The parabolic behaviour of the DMF-water interaction energy depends on the number of species of each component making up the mixture. Comparing the water-DMF interaction energy with similar data obtained from other binary aqueous mixtures previously studied by us, like tetrahydrofuran [7] and acetone [10,26], which also contain oxygen in the molecular formulas, one notices that the water-DMF interaction is stronger. Therefore, in the water-DMF mixture the balance between disruption/formation of hydrogen bonds is an important component of the energetics of the mixing process.

\subsection{Radial Distribution Functions}

The water-DMF radial distribution functions of an equimolar solution are shown in Figure 3. The two sharp peaks on the $\mathrm{O}_{d} / \mathrm{H}_{\mathrm{w}}$ and $\mathrm{O}_{\mathrm{d}} / \mathrm{O}_{\mathrm{w}}$ correlation functions indicate hydrogen-bonding formation between the oxygen site of DMF and hydrogen of water. $\mathrm{O}_{\mathrm{d}} / \mathrm{H}_{\mathrm{w}}$ exhibits two peaks (at $r=1.9$ and $3.2 \AA$ ) indicating that just one hydrogen site of a water molecule is closely related to the hydrogen-bond formation between water and DMF. This observation is corroborated by the fact that the separation between these two peaks is approximately equal to the $\mathrm{H}-\mathrm{H}$ distance in the water molecule. The integration of the $\mathrm{O}_{\mathrm{d}} / \mathrm{H}_{\mathrm{w}}$ correlation function over the first peak, as discussed above, gives a value of $\approx 1$, characterising the formation of water-DMF dimers. A weaker correlation between methyl groups and water oxygen is also observed near $r=3.6 \AA$, indicating a preferential conformation for the water-DMF dimer where the oxygen of the water molecule is facing a methyl group of DMF.

Figure 4 show the dimer between DMF and water obtained by optimisation at MM2 level [27]. The site-site distances calculated from this dimer geometry reproduce strictly the features observed in the radial distribution functions, $g(r)$, obtained for the mixture. The site-site correlation functions obtained for a pure liquid and this liquid in a binary mixture can be compared. It is verified that the formation of water-DMF dimer decreases the correlation between methyl and oxygen sites of DMF compared with the pure liquid [9]. Finally, as one can notice in the Fig. 3, a structureless correlation function was obtained for the hydrogen atom bounded to DMF carbonyl and the

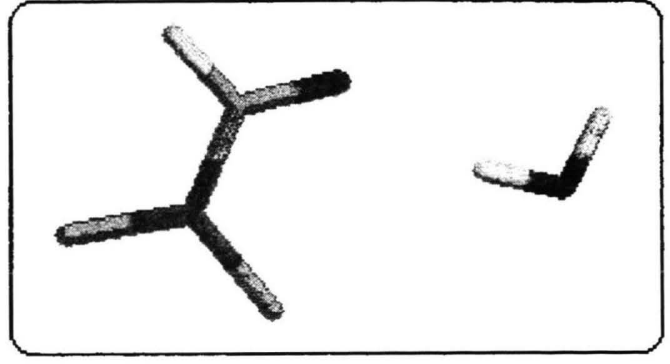

Fig. 4. The dimer DMF-water optimised at the MM2 level. Geometric parameters: $r\left(\mathrm{O}_{\mathrm{DMF}}-\mathrm{H}_{\mathrm{W}}\right)=1.93,3.10 \AA$, $r\left(\mathrm{O}_{\mathrm{DMF}}-\mathrm{O}_{\mathrm{W}}\right)=2.86 \AA, r\left(\mathrm{Me}(\mathrm{cis})-\mathrm{O}_{\mathrm{W}}\right)=3.48 \AA, \angle(\mathrm{CO}-$ $\left.\mathrm{H}_{\mathrm{W}}\right)=132^{\circ}, \angle\left(\mathrm{O}_{\mathrm{DMF}}-\mathrm{H}_{\mathrm{W}}-\mathrm{O}_{\mathrm{W}}\right)=163^{\circ}, \angle\left(\mathrm{O}_{\mathrm{DMF}}-\mathrm{H}_{\mathrm{W}}-\mathrm{O}_{\mathrm{W}^{-}}\right.$ $\left.\mathrm{H}_{\mathrm{W}}\right)=-24^{\circ}$.

oxygen site of water molecules. Therefore, the interaction $\mathrm{H}-\mathrm{O}$ is important to stabilise the dimers of DMF in pure liquid [9], but it is irrelevant in the formation of DMF-water dimers. This result can be explained by comparing the partial charges associated with the hydrogen and oxygen sites in DMF $\left(q_{\mathrm{H}}=0.12 \mathrm{e} q_{\mathrm{O}}\right.$ $=-0.50)$ with those ones in TIP4P water $\left(q_{\mathrm{H}}=0.52\right.$ and $\left.q_{\mathrm{O}}=-1.04\right)$. Due to the lower partial charge on the hydrogen site of DMF, breaking an $\mathrm{H}_{\mathrm{W}}-\mathrm{O}_{\mathrm{W}}$ association to form an $\mathrm{H}_{\mathrm{DMF}}-\mathrm{O}_{\mathrm{W}}$ one is not energetically competitive.

\subsection{Solvent Effects}

The influence of the solvent on the rotational energy barrier between cis and trans conformers of $\mathrm{N}$ methylacetamyde and $\mathrm{N}$-methylformamyde in water have been studied by Jorgensen and Gao [28] using SPT. Similar studies using different methodologies have been reported by other authors [29-31]. In the present work, hydration effects on the rotational energy barrier around the peptide bond of the DMF molecule are investigated. The chain O-C-N$\mathrm{C}$ has been chosen to define the dihedral angle (see Figure 4). The free energy contribution from solutesolvent interaction was calculated using (1), and an increment $\Delta \phi=9^{\circ}$ was used to perform the rotation around the peptide bond. All potential parameters remain fixed throughout the calculations. Results obtained for the hydration free energy as a function of the rotation angle are shown in Table 1.

The results show a very small influence of hydration effects on the free energy difference as a function of the rotation angle. The free energy decreases by $\approx 1.0 \mathrm{kcal} / \mathrm{mol}$ when the dihedral angle goes from $0^{\circ}$ 
Table 1. Free energy (in $\mathrm{kcal} / \mathrm{mol}$ ) for cis-trans DMF interconvertion in water obtained by SPT.

\begin{tabular}{rrrr}
\hline$\phi_{i}$ & $\phi_{j}$ & $\Delta G(i \rightarrow j)$ & $\Delta G_{\text {total }}$ \\
\hline 0 & 9 & $0.03 \pm 0.21$ & 0.03 \\
9 & 18 & $-0.02 \pm 0.16$ & 0.01 \\
18 & 27 & $-0.9 \pm 0.20$ & -0.08 \\
27 & 36 & $-0.15 \pm 0.21$ & -0.23 \\
36 & 45 & $-0.20 \pm 0.19$ & -0.43 \\
45 & 54 & $-0.15 \pm 0.12$ & -0.58 \\
54 & 63 & $-0.20 \pm 0.10$ & -0.78 \\
63 & 72 & $-0.12 \pm 0.11$ & -0.90 \\
72 & 81 & $-0.06 \pm 0.20$ & -0.96 \\
81 & 90 & $-0.06 \pm 0.17$ & $-1.02 \pm 0,54$ \\
\hline
\end{tabular}

Table 2. Solute-solvent interaction energy (in $\mathrm{kcal} / \mathrm{mol}$ ) as a function of the rotation angle around the peptide bond in DMF molecule.

\begin{tabular}{ccccccc}
\hline$\phi\left(^{\circ}\right)$ & 0 & 9 & 18 & 27 & 36 & 45 \\
$-\mathrm{E}$ & 27.25 & 26.92 & 27.11 & 26.83 & 27.71 & 27.69 \\
\hline$\phi\left({ }^{\circ}\right)$ & 54 & 63 & 72 & 81 & 90 & \\
$-\mathrm{E}$ & 27.98 & 28.08 & 28.51 & 28.43 & 28.51 & \\
\hline
\end{tabular}

to $90^{\circ}$. Jorgensen and Gao [28] have obtained a value of $\Delta G=-1.35 \mathrm{kcal} / \mathrm{mol}$ for the free energy of rotation of the $\mathrm{N}$-methylacetamyde molecule from 0 to $90^{\circ}$. This result is very close to $\Delta G=-1.02 \mathrm{kcal} / \mathrm{mol}$, the value obtained in the present work for rotation of DMF in the same angular range. Consequently, the average energy for solute-solvent interaction is almost independent of the rotation angle, as is reported in Table 2. Since $\Delta G=\Delta H-T \Delta S$, a large change in the free energy of solvation as function of the rotation angle could be possible due to an expressive contribution of the entropic term $-T \Delta \mathrm{S}$. Such a contribution should be associated with a large solvent reorganisation in the neighbourhood of the solute molecule. The data in Table 3 show that this is not the case, indicating that the changes in the solvent organisation are not sufficient to stabilise the DMF molecule in a not-planar configuration. Molecular Mechanics calculations in vacuo show that the height of the rotational barrier of DMF is near 20 $\mathrm{kcal} / \mathrm{mol}$ at $\phi=90^{\circ}$. The difference in the solutesolvent interaction energy between the two conformers was nearly $-1.26 \mathrm{kcal} / \mathrm{mol}$, and the free energy difference between them was $-1.02 \mathrm{kcal} / \mathrm{mol}$. This close agreement can be explained by considering that in (1) the numerical approximation
Table 3. Average water-water interaction energy $\left(E_{\mathrm{ww}}\right)$ in water-DMF solution as a function of the rotation angle on the peptide bond.

\begin{tabular}{lrrrrr}
\hline$\phi\left(^{\circ}\right)$ & 9 & \multicolumn{1}{c}{27} & \multicolumn{1}{c}{45} & \multicolumn{1}{c}{63} & \multicolumn{1}{c}{81} \\
\hline$-E_{\mathrm{ww}}$ & 10.77 & 10.75 & 10.71 & 10.80 & 10.76 \\
$-\left(E_{\mathrm{ww}}-E_{\mathrm{ww}}^{0}\right)$ & 0.72 & 0.70 & 0.66 & 0.75 & 0.71 \\
\hline
\end{tabular}

Energy in $\mathrm{kcal} / \mathrm{mol}$. $E_{\mathrm{ww}}^{0}=-10.05 \mathrm{kcal} / \mathrm{mol}$, the average waterwater interaction energy in pure water.

$$
\Delta G=-k_{\mathrm{B}} T \ln \langle\exp (-\beta \Delta E)\rangle \approx\langle\Delta E\rangle
$$

holds when $\Delta E$, the value of perturbation energy, is almost the same for every configuration generated along the Monte Carlo random walk. In this case, the free energy difference is very close to the contribution from the mechanic energy. Thus the contribution from the entropic term $-T \Delta S$ is nearly zero.

\section{Conclusions}

The results obtained in this work indicate strong interaction between water and DMF molecules in aqueous solutions of DMF. The excess energies calculated for the water-DMF mixing process are in fair accord with experimental data. This finding corroborates the usefulness of combing rules to obtain potential parameters for cross interaction in liquid simulations. The main contribution to the water-DMF interaction energy comes from the oxygen site of DMF and the hydrogen sites of water, which leads to the formation of hydrogen-bonded dimers. This interaction plays an important role in the stabilisation of the solute and emphasises the importance of the water-carbonyl group interaction in the stabilisation of solutes containing this group. Investigation of hydration effects on the stabilisation of the DMF molecule in aqueous solution shows the usefulness of SPT methodology to study solvent effects. It can provide a great number of interesting data on the solvation process. In the present work, the influence of the hydration process on the rotational energy barrier of DMF was found to be negligible, as the energetic changes due to hydration are not strong enough to stabilise another configuration of DMF than the planar one. Therefore, the rigidity of the gas phase peptide bond is not disturbed by hydration. 
[1] J. Åqvist, J. Comp. Chem. 17, 1587 (1996).

[2] J. Åqvist, C. Medina, and J. E. Samuelson, Protein Eng. 7, 385 (1994).

[3] D. W. Hermann, Computer Simulation Methods, Springer-Verlag, Berlin 1986.

[4] E. R. Davidson, Chem. Rev. 93, 2337 (1993).; C. E. Dykstra, idem, 93, 2339 (1993).; R.S. Berry, idem, 93, 2379 (1993).

[5] B. M. Ladanyi and M. S. Skaf, Rev. Phys. Chem. 44, 335 (1993).

[6] Y. M. Kessler, Y. P. Puhovski, M. G. Kiselev, and I. I. Vaisman, in Chemistry of Non Aqueous Solutions: Current Progress, G. Mamantov and A. I. Popov, Eds, VHC, New York 1994.

[7] L. C. G. Freitas and J. M. M. Cordeiro, J. Mol. Struct. (Theochem). 335, 189 (1995).

[8] A. L. L. Sinoti, J. R. S. Politi, and L. C. G. Freitas, J. Braz. Chem. Soc. 7, 133 (1996).

[9] J. M. M. Cordeiro, Int. J. Quant. Chem. 65, 709 (1997).

[10] L. C. G. Freitas, J. M. M. Cordeiro, and F. L. L. Garbujo, J. Mol. Liq. 79, 1 (1999).

[11] R. W. Zwanzig, J. Chem. Phys. 22, 1420 (1954).

[12] P. A. Kollman and K. M. Merz Jr., Acc. Chem. Res. 23, 246 (1990).

[13] T. P. Straatsma and J. A. McCammon, Annu. Rev. Phys. Chem. 43, 407 (1992).

[14] D. A. Pearlm, J. Phys. Chem. 98, 1487 (1994).

[15] H. A. Carlson, T. B. Nguyen, M. Orozco, and W. L. Jorgensen, J. Comp. Chem. 14, 1240 (1995).
[16] M. P. Allen and D. J. Tildesley, Computer Simulation of Liquids, Oxford Express, Oxford 1987.

[17] W. L. Jorgensen, J. Chandrasekhar, J. D. Madura, R. W. Impey, and M. L. Klein, J. Chem. Phys. 79, 926 (1983).

[18] N. Metropolis, A. Rosembluth, M. N. Rosembluth, A. Teller, and E. Teller, J. Chem. Phys. 21, 1087 (1953).

[19] L. C. G. Freitas, DIADORIM program, Dept. of Chemistry, University Federal of SÔo Carlos, SP, Brazil.

[20] J. M. M. Cordeiro, Quimica Nova 21, 698 (1998).

[21] M. Neumann, Mol. Phys. 50, 841 (1983).

[22] L. C. G. Freitas, submitted for publication.

[23] M. Cilense, A. V. Benedetti, and D. R. Vollet, Therm. Acta 63, 151 (1983).

[24] G. E. Rodgers and R. A. Plane, J. Chem. Phys. 63, 818 (1975)

[25] J. N. Spencer, J. Phys. Chem. 85, 1236 (1981).

[26] E. C. Meng, J. C. Caldwell, and P. A. Kolmann, J. Phys. Chem. 100, 2367 (1996).

[27] PCModel, Serena Software, Bloomington, USA.

[28] W. L. Jorgensen and J. Gao, J. Amer. Chem. Soc. 107, 4212 (1988).

[29] D. L. Beveridge and F. M. DiCapua, Ann. Rev. Biophys. Byophys. Chem. 18, 431 (1989).

[30] B. Jayaram, M. Mezei, and D. L. Beveridge, J. Amer. Chem. Soc. 110, 1691 (1988).

[31] M. Mezei, P. K. Mehrotra, and D. L. Beveridge, J. Amer. Chem. Soc. 107, 2239 (1985). 